\title{
Oscillatory Behavior of the Rate of Escape through an Unstable Limit Cycle
}

\author{
Robert S. Maier ${ }^{(1)}$ and D. L. Stein ${ }^{(2)}$ \\ Mathematics $^{(1)}$ and Physics ${ }^{(2)}$ Departments, University of Arizona, Tucson, Arizona 85721
}

\begin{abstract}
Suppose a two-dimensional dynamical system has a stable attractor that is surrounded by an unstable limit cycle. If the system is additively perturbed by white noise, the rate of escape through the limit cycle will fall off exponentially as the noise strength tends to zero. By analysing the associated Fokker-Planck equation we show that in general, the weaknoise escape rate is non-Arrhenius: it includes a factor that is periodic in the logarithm of the noise strength. The presence of this slowly oscillating factor is due to the nonequilibrium potential of the system being nondifferentiable at the limit cycle. We point out the implications for the weak-noise limit of stochastic resonance models.

PACS numbers: 02.50.-r, 05.40.+j
\end{abstract}

A particularly interesting phenomenon is the occurrence of noise-induced transitions between attractors of a dynamical system. Such transitions occur in chemical physics, where the transition is a motion across a transition-state surface from a reactant region to a product region. They also occur in statistical physics, and in other fields where stochastic modelling plays a role [1,2.

If the noise is white, or has a short correlation time and may be approximated as white, then the probability density of the system will satisfy a Fokker-Planck equation. This equation governs the way in which noise-induced transitions occur. By the 'rate' at which a specified transition takes place we shall mean the probability that it occurs, per unit time. At least in finite-dimensional systems, any such rate should fall off exponentially as $\epsilon$, the noise strength, tends to zero. (In thermal applications $\epsilon$ would be proportional to $k T$.) In fact, each transition should be characterized by an activation energy $\Delta W$, with the transition rate falling off to leading order as $e^{-\Delta W / \epsilon}$. Computing the pre-exponential factor requires a careful analysis of the Fokker-Planck equation [2 [5].

Most work has focused on the case when the competing attractors of the dynamical system are separated by a separatrix (i.e., a 'ridge') containing a saddle point. However, models where the separatrix is instead an unstable limit cycle arise in the context of chemical reactions constrained to occur far from equilibrium [6]. Also, transitions across an unstable limit cycle separating steady states of periodic vibration occur in models of stochastic resonance in bistable continuous systems [7]8]. A full analysis of noise-driven escape through an unstable limit cycle accordingly seems called for.

Previous work on noise-driven transitions in models with an unstable limit cycle is found in Refs. [2, 9-13. Graham and Tél [9, 10, and Day 11, 13, have noted that the nonequilibrium potential $W$, as a function on the state space of the system being modelled, will be nondifferentiable ('wild') near the limit cycle if the system fails to satisfy a form of detailed balance. Naeh et al. [2] began an analysis of the Fokker-Planck equations associated to such models, by a method of matched asymptotic expansions, but their analysis assumed that $W$ was differentiable at the limit cycle.

In this Letter we begin an asymptotic analysis of the rate of escape through an unstable limit cycle that incorporates the insights of Graham and Tél, and of Day, and obtain a striking result. We show that generically, in two-dimensional models with an unstable limit cycle enclosing an attractor, the rate of escape $R$ is given by a non-Arrhenius formula of the form

$$
R \sim \text { const } \times \epsilon^{b} G(|\log \epsilon|) e^{-\Delta W / \epsilon}
$$

in the weak-noise $(\epsilon \rightarrow 0)$ limit. Here $b$ is modeldependent, and the factor $G(|\log \epsilon|)$ is a modeldependent periodic function of $|\log \epsilon|$. The presence of such slowly oscillating factors in the expressions for noise-dependent transition rates has not previously been suspected. It indicates that even in bistable dynamical systems with effective dimensionality as low as two, relaxation phenomena may be more complicated than is commonly believed. Our analysis applies whenever the system is truly two-dimensional, i.e., is nonseparable.

Models.-We consider models with dynamics that are those of a Brownian particle moving in a drift field, i.e.,

$$
\dot{x}^{i}=u^{i}(\boldsymbol{x})+\epsilon^{1 / 2} \sum_{\alpha=1}^{2} \sigma^{i}{ }_{\alpha}(\boldsymbol{x}) \eta_{\alpha}(t) .
$$

Here $\boldsymbol{x}=\left(x^{1}, x^{2}\right)$ is a pair of state variables, and the drift field $\boldsymbol{u}=\left(u^{1}, u^{2}\right)$ specifies the dynamics in the absence of noise. $\left(\eta_{1}, \eta_{2}\right)$ is a pair of white noise processes, satisfying $\left\langle\eta_{\alpha}(s) \eta_{\beta}(t)\right\rangle=\delta_{\alpha \beta} \delta(s-t) . \boldsymbol{\sigma}=\left(\sigma^{i}{ }_{\alpha}\right)$ is a socalled noise matrix that is allowed to be state-dependent (a 'zweibein' field). The probability density $\rho=\rho(\boldsymbol{x}, t)$ of such a system satisfies the Fokker-Planck equation

$$
\dot{\rho}=-\mathcal{L}_{\epsilon}^{*} \rho \equiv(\epsilon / 2) \partial_{i} \partial_{j}\left[D^{i j}(\boldsymbol{x}) \rho\right]-\partial_{i}\left[u^{i}(\boldsymbol{x}) \rho\right],
$$

where the diffusion tensor $\boldsymbol{D}=\left(D^{i j}\right) \equiv \boldsymbol{\sigma} \boldsymbol{\sigma}^{t}$. The operator $\mathcal{L}_{\epsilon}^{*}$ is the (forward) Fokker-Planck operator. We consider here the case when there is a point attractor $S$ in the $\left(x^{1}, x^{2}\right)$-plane, with domain of attraction $\Omega$, for which the boundary $\partial \Omega$ is an unstable limit cycle.

This framework is sufficiently general that it can accomodate two-dimensional models with overdamped dynamics, or one-dimensional models with underdamped 


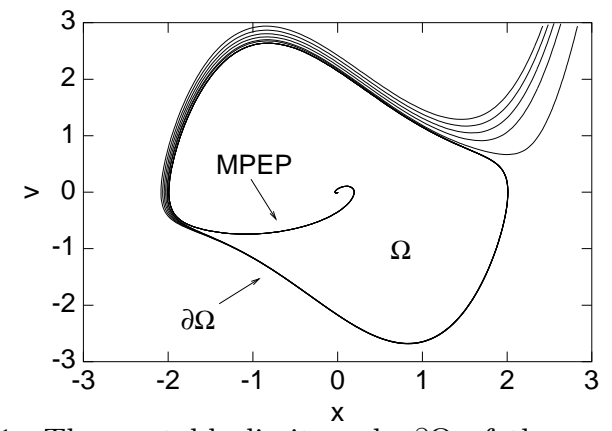

FIG. 1. The unstable limit cycle $\partial \Omega$ of the van der Pol model, and the MPEP, which emerges from the attractor $(0,0)$ and spirals into it. The trajectories exiting from $\Omega$ are optimal trajectories that are perturbations of the MPEP.

dynamics. In the latter case one of the state variables $\left(x^{1}\right.$, say) would be a position, and the other a velocity. Our simulations below are of a model of this sort, namely

$$
\begin{aligned}
& \dot{x}=v \\
& \dot{v}=-x+\left(v^{2}-1\right) v+\epsilon^{1 / 2} \eta_{2}(t) .
\end{aligned}
$$

This is a time-reversed van der Pol oscillator, the asymptotic analysis of which was begun by Day [13]. Here $\left(x^{1}, x^{2}\right)=(x, v), S=(0,0)$, and $\boldsymbol{D}=\operatorname{diag}(0,1)$ is degenerate. The unstable limit cycle is shown in Fig. 1.

The Analysis.-To estimate the rate of escape through $\partial \Omega$, we use the Kramers flux-over-the-barrier technique 14. Suppose that escaping Brownian particles are re-injected at the attractor $S$, and a steady state has been reached. The probability density in this state, which we denote $\rho_{0}$, will satisfy $\mathcal{L}_{\epsilon}^{*} \rho_{0}=0$. When the noise strength $\epsilon$ is small, $\rho_{0}$ will be tightly peaked near $S . \rho_{0}(\boldsymbol{x})$, at points $\boldsymbol{x}$ on the limit cycle $\partial \Omega$ and outside it, will be suppressed by a factor $\sim e^{-\Delta W / \epsilon}$ relative to $\rho_{0}(S)$. Since the Fokker-Planck equation has the form of a continuity equation, with current density $J^{i}[\rho]$ equalling $\rho u^{i}-(\epsilon / 2) \partial_{j}\left[D^{i j} \rho\right]$, the escape rate $R$ may be computed as the flux of probability through $\partial \Omega$, i.e.,

$$
R \sim \int_{\partial \Omega} \boldsymbol{J}\left[\rho_{0}\right] \cdot \boldsymbol{n} d \ell / \int_{\Omega} \rho_{0} d^{2} x .
$$

Here $\boldsymbol{n}$ denotes the outward normal on $\partial \Omega$.

To derive the oscillating formula of eq. (1) from eq. (6), we introduce a WKB approximation to the steady-state density $\rho_{0}$ in the weak-noise limit [2]5]. We write

$$
\rho_{0}(\boldsymbol{x}) \sim K(\boldsymbol{x}) \exp (-W(\boldsymbol{x}) / \epsilon) .
$$

Here $W(\boldsymbol{x})$ is an 'activation energy' controlling noiseinduced fluctuations from the attractor to the vicinity of $\boldsymbol{x}$. Though (7) resembles a Maxwell-Boltzmann distribution, $W$ is a nonequilibrium potential, since the steady state is not necessarily an equilibrium state, in that it does not necessarily satisfy detailed balance. We set $W(S)=0$, so that $\Delta W$, the falloff rate of the escape rate, is the value of $W$ attained on the unstable limit cycle.
Substituting (7) into $\mathcal{L}_{\epsilon}^{*} \rho_{0}=0$ and separating out the $\mathcal{O}\left(\epsilon^{-1}\right)$ terms yields the eikonal equation

$$
H\left(x^{i}, \partial W / \partial x^{i}\right)=0
$$

where

$$
H\left(x^{i}, p_{i}\right) \equiv \frac{1}{2} D^{i j}(\boldsymbol{x}) p_{i} p_{j}+u^{i}(\boldsymbol{x}) p_{i}
$$

is a so-called Wentzell-Freidlin Hamiltonian 15. Equation (8) has the form of a Hamilton-Jacobi equation, with $W$ a classical action at zero energy. To compute $W(\boldsymbol{x})$ one may simply use Hamilton's equations of motion to generate the zero-energy classical trajectory from $S$ to $\boldsymbol{x}$. The quantity $W(\boldsymbol{x})$ will necessarily equal $\int \boldsymbol{p} \cdot d \boldsymbol{x}$, the line integral being taken from $S$ to $\boldsymbol{x}$ along the trajectory. We stress that $\boldsymbol{p}=\boldsymbol{\nabla} W$ here is not a physical momentum: it is a mathematical artifact. Hamilton's equation $\dot{x}^{i}=D^{i j} p_{j}+u^{i}$ reveals that $\boldsymbol{p}$ measures the extent to which the classical trajectories move against the drift $\boldsymbol{u}$. Deterministic (no-noise) trajectories have $\boldsymbol{p} \equiv \mathbf{0}$.

By separating out the $\mathcal{O}\left(\epsilon^{0}\right)$ terms in $\mathcal{L}_{\epsilon}^{*} \rho_{0}=0$ one can show that the pre-exponential factor $K(\boldsymbol{x})$ satisfies [2, []

$$
\dot{K}=-\left(\boldsymbol{\nabla} \cdot \boldsymbol{u}+D^{i j} W_{, i j} / 2\right) K,
$$

the time derivative being a derivative with respect to transit time along the zero-energy classical trajectory. Here $W_{, i j} \equiv \partial W / \partial x^{i} \partial x^{j}$. By differentiating the Hamilton-Jacobi equation one can show that the matrix $\left(W_{, i j}\right)$ satisfies a Riccati equation along the trajectory:

$$
\dot{W}_{, i j}=-D^{k l} W_{, k i} W_{, l j}-u^{k}{ }_{, i} W_{, k j}-u^{k}{ }_{, j} W_{, k i}-u_{, i j}^{l} p_{l} .
$$

This facilitates the computation of $K$.

The zero-energy classical trajectories emanating from the attractor, sometimes called optimal trajectories, have a direct physical interpretation: they are the most probable fluctuational trajectories. If a noise-induced fluctuation from $S$ to $\boldsymbol{x}$ occurs, in the limit of weak noise it should occur with increasing likelihood along an optimal trajectory terminating at $\boldsymbol{x}$. Such trajectories have been seen experimentally [16]. In the weak-noise limit the most probable escape path (MPEP) will be the leastaction optimal trajectory extending from $S$ to the limit cycle $\partial \Omega$. Normally this trajectory will spiral into $\partial \Omega$, rather than crossing $\partial \Omega$ in finite time, for the following reason. If the MPEP crossed the limit cycle, the crossing point would be a 'hot spot' through which escape would preferentially occur. The tangential derivative $\partial_{t} W$ (i.e., the tangential momentum $p_{t}$ ) would necessarily be zero there. But the normal drift velocity $u^{n}$ equals zero on $\partial \Omega$. So the second term in eq. (9) would vanish at the hotspot. If $\boldsymbol{D}$ is nondegenerate, eqs. (8)-(9) imply that $\boldsymbol{p}=\mathbf{0}$ there, i.e., $\dot{\boldsymbol{x}}=\boldsymbol{u}$. That is, the ostensible MPEP would be a deterministic trajectory, which 
is impossible. The MPEP normally spirals into the unstable limit cycle even when $\boldsymbol{D}$ is degenerate. In Fig. 1 we show the MPEP of the van der Pol model (仼)-(5).

Multivaluedness.-Dykman, Millonas, and Smelyanskiy [17] and the present authors [4] have stressed that $W$ and $K$ may be multivalued functions of the system state $\boldsymbol{x}$, since any given point $\boldsymbol{x}$ may be the endpoint of more than one optimal trajectory. This normally happens in models with an unstable limit cycle, as Fig. 1 shows. Optimal trajectories that are perturbations of the MPEP do not spiral into the limit cycle. Rather, they approach it, and wind around the region $\Omega$ a number of times, all the while deviating farther from the MPEP. They eventually exit from $\Omega$ (if the perturbation is in the outward direction) or move back toward the attractor (if the perturbation is inward). As a consequence, any point $\boldsymbol{x}$ near the unstable limit cycle is the endpoint of any of an infinite, discrete set of optimal trajectories, which differ from each other in their winding number $l$, which may be arbitrarily large. $W$ and $K$ are infinitevalued, and the WKB approximation (7) generalizes to

$$
\rho_{0}(\boldsymbol{x}) \sim \sum_{l} K^{(l)}(\boldsymbol{x}) \exp \left(-W^{(l)}(\boldsymbol{x}) / \epsilon\right) .
$$

In the weak-noise limit, this sum is dominated by the term with minimum $W^{(l)}(\boldsymbol{x})$. Equivalently, fluctuations to any point $\boldsymbol{x}$ in $\Omega$, in the limit of weak noise, proceed preferentially along the physical optimal trajectory from the attractor to $\boldsymbol{x}$ : the least-action one. Subdominant trajectories contribute at larger noise strengths, however.

We shall use (12) to compute the flux of Brownian particles over the barrier $\partial \Omega$. We first approximate $W^{(l)}$ and $K^{(l)}$ near $\partial \Omega$ by extending the results of Naeh et al. [2]. As a first attempt, suppose that $W$ is singlevalued and (to leading order) quadratic near $\partial \Omega$, so that it can be approximated as $\Delta W+W_{, n n} n^{2} / 2$. Here $n$ is the distance in from $\partial \Omega$, in the normal direction, and the second normal derivative $W_{, n n}=\partial p_{n} / \partial n<0$ depends on position along $\partial \Omega$. The matrix equation (11) yields

$$
\dot{W}_{, n n}=-D^{n n}\left(W_{, n n}\right)^{2}-2 u^{n}{ }_{, n} W_{, n n} \quad,
$$

a Riccati equation along $\partial \Omega$. The final term in (11) has dropped out, as $\boldsymbol{p}=\boldsymbol{\nabla} W$ is zero on $\partial \Omega$ if $W$ behaves quadratically there. $W_{, n n}$ as a function of position along $\partial \Omega$ may be computed from (13) by integration [2].

A problem with this approach was pointed out by Graham and Tél [9,10]. Assuming that $W$ is single-valued near the unstable limit cycle is much the same as assuming that optimal trajectories that are perturbations of the MPEP, as well as the MPEP itself, spiral into the limit cycle. What actually happens near $\partial \Omega$ is revealed by a Poincaré section. Suppose we choose some point on $\partial \Omega$, and plot the pair $\left(n, p_{n}\right)$, i.e., normal displacement and normal momentum, for each optimal trajectory that passes nearby. If $W$ were quadratic in $n$, i.e.,

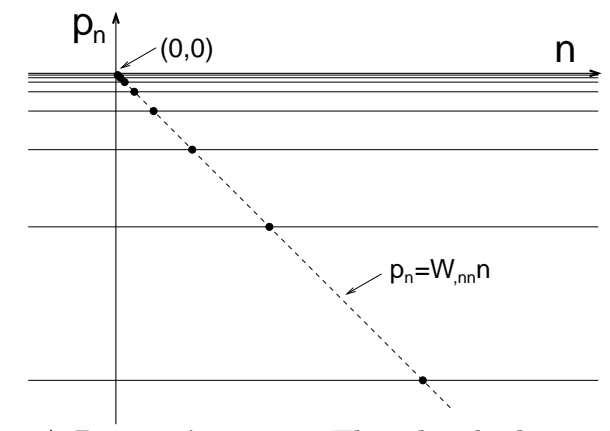

FIG. 2. A Poincaré section. This sketch shows the points $\left(n, p_{n}\right)$ generated by the optimal trajectories passing by some specified point on $\partial \Omega$. The dots are generated by the MPEP, spiralling into $\partial \Omega$. Cf. Figs. 1-3 of Graham and Tél [10].

$p_{n}=\partial W / \partial n$ were linear in $n$, the points $\left(n, p_{n}\right)$ would lie on a line with slope $W_{, n n}$ passing through $(0,0)$. What happens instead is shown in Fig. 2. The MPEP generates points that tend to $(0,0)$ geometrically, and lie along the dashed line $p_{n}=W_{, n n} n$. But perturbations of it generate points that lie along the horizontal solid lines.

Figure 2 can be interpreted in terms of a 'return map' that updates $\left(n, p_{n}\right)$ whenever an optimal trajectory winds once around $\Omega$. This map will have $(0,0)$ as fixed point. For the MPEP to spiral into $\partial \Omega$ and yield points along the ideal line, the linearized return map at $(0,0)$ must have $\left(1, W_{, n n}\right)$ as an eigenvector, with eigenvalue less than 1 . And since deterministic $(\boldsymbol{p} \equiv \mathbf{0})$ trajectories that 'peel off' from $\partial \Omega$ do so geometrically, $(1,0)$ must also be an eigenvector, with eigenvalue greater than 1. By Liouville's Theorem these eigenvalues must be reciprocals, so we denote them $c^{-1}$ and $c$. With each turn, the MPEP decreases its distance from $\partial \Omega$ by a factor $c$, and deterministic trajectories that diverge from $\partial \Omega$ increase their distance from it by a factor $c$.

Figure 2 can now be explained. Suppose the MPEP intersects the $\left(n, p_{n}\right)$ plane at $a\left(1, W_{, n n}\right)$. Optimal trajectories that are small perturbations of the MPEP will intersect it at $a\left(1, W_{, n n}\right)+\lambda \boldsymbol{v}$, where $\lambda$ is the perturbation strength and $\boldsymbol{v}$ is model-dependent. We write $\boldsymbol{v}=\alpha_{s}\left(1, W_{, n n}\right)+\alpha_{u}(1,0)$, where $\alpha_{s}, \alpha_{u} \neq 0$ in general. By iterating the return map, we see that after winding $l$ more times, the trajectories intersect the $\left(n, p_{n}\right)$ plane at

$$
a c^{-l}\left(1, W_{, n n}\right)+\lambda \alpha_{u} c^{l}(1,0) .
$$

The $\alpha_{s}$ term has been dropped here, since it becomes negligible with respect to the $\alpha_{u}$ term as $l \rightarrow \infty$. It is the second term in (14) that gives rise to the horizontal solid lines of Fig. 2, as $\lambda$ is varied away from zero.

On the MPEP, the nonequilibrium potential $W$ behaves quadratically near $\partial \Omega$. In particular, at $n=a c^{-l}$, $W \approx \Delta W+W_{, n n}\left(a c^{-l}\right)^{2} / 2$. It follows that the l'th value $W^{(l)}(n)$ of the infinite-valued function $W(n)$, which arises from trajectories that wind $l$ times around $\partial \Omega$, is

$$
W^{(l)}(n) \approx W\left(a c^{-l}\right)+(\partial W / \partial n)\left(n-a c^{-l}\right)
$$




$$
\begin{aligned}
& =\Delta W+W_{, n n}\left(a c^{-l}\right)^{2} / 2+a c^{-l} W_{, n n}\left(n-a c^{-l}\right) \\
& =\Delta W+W_{, n n}\left(a c^{-l} n-a^{2} c^{-2 l} / 2\right)
\end{aligned}
$$

Each $W^{(l)}$, as a function of the normal distance $n$, is to leading order linear, not quadratic. This has been noticed by Graham and Tél [9, 10, who also noted that if one plots the physical (i.e., minimum) value of $W(n)$, one obtains a piecewise linear approximation to the ideal parabola $\Delta W+W_{, n n} n^{2} / 2$. The physical $W$ is nondifferentiable at a sequence of points converging to $n=0$.

Oscillatory Asymptotics.-To apply the Kramers method, we need the prefactors $K^{(l)}$, as well as $W^{(l)}$. At any $\boldsymbol{x}, K^{(l)}$ is computed by integrating eq. (10) along an optimal trajectory that winds $l$ times around $\Omega$, and terminates at $\boldsymbol{x}$. We must distinguish here between the 'ideal' $W_{, n n}$, which is a mathematical abstraction (the periodic solution of the Riccati equation (13)), and the actual second derivatives $\partial^{2} W^{(l)} / \partial x^{i} \partial x^{j}$. It is the latter that appear in (10). In both (15) and Fig. 2, which were computed on the basis of the linearized return map, $\partial^{2} W^{(l)} / \partial n^{2} \equiv 0$, and hence $\partial^{2} W^{(l)} / \partial x^{i} \partial x^{j} \equiv 0$, for every $l$. Keeping higher-order terms would keep the second derivatives $\partial^{2} W^{(l)} / \partial x^{i} \partial x^{j}$ from being identically zero, but they would still fall to zero as $\partial \Omega$ is approached.

It follows that when computing $K$ near $\partial \Omega$, we may replace $(10)$ by $\dot{K} \approx-(\boldsymbol{\nabla} \cdot \boldsymbol{u}) K$. In the limit of large winding number $l$, which involves integration along a trajectory that spirals ever closer to $\partial \Omega$, this yields $K^{(l+1)} / K^{(l)} \sim \exp [-\oint(\boldsymbol{\nabla} \cdot \boldsymbol{u}) d t]$, the integral being taken once around $\partial \Omega$. By examination, this limiting quotient equals $c^{-1}$. We shall write $K^{(l)} \approx A c^{-l}$, where $A$ is a function of position along $\partial \Omega$. This $n$-independent approximation is increasingly accurate as $n \rightarrow 0$.

Substituting the approximations for $K^{(l)}$ and $W^{(l)}$ into (12) yields an approximation to the steady-state probability density near the unstable limit cycle, i.e.,

$\rho_{0}(n) \sim A e^{-\Delta W / \epsilon} \sum_{l} c^{-l} \exp \left\{-W_{, n n}\left[a c^{-l} n-a^{2} c^{-2 l} / 2\right] / \epsilon\right\}$

(Recall that $W_{, n n}<0$.) It follows that $\boldsymbol{J}\left[\rho_{0}\right] \cdot \boldsymbol{n}$, the normal component of the probability flux density at $n=0$ (i.e., through $\partial \Omega$ ), is to leading order

$$
\text { const } \times e^{-\Delta W / \epsilon} \sum_{l} c^{-2 l} \exp \left(-a^{2}\left|W_{, n n}\right| c^{-2 l} / 2 \epsilon\right) .
$$

The sum over winding number $l$ may be approximated by a discrete analogue of Laplace's method. As $\epsilon \rightarrow 0$, the dominant terms in the sum have $l \approx|\log \epsilon| / 2 \log c$. Let $l^{*}$ be the greatest integer less than or equal to $|\log \epsilon| / 2 \log c$, and let $h \equiv|\log \epsilon| / 2 \log c-l^{*}$. Changing the summation variable to $k=l-l^{*}$ allows one to approximate $\boldsymbol{J}\left[\rho_{0}\right] \cdot \boldsymbol{n}$ in the $\epsilon \rightarrow 0$ limit, up to a constant factor, by

$$
\epsilon^{q} e^{-\Delta W / \epsilon} \sum_{k=-\infty}^{\infty} c^{-2(k-h)} \exp \left(-a^{2}\left|W_{, n n}\right| c^{-2(k-h)} / 2\right) .
$$

Here $q \equiv(1+2 \log c) / 2$, and the summation is periodic in $h$ with period unity. Equivalently, the summation is periodic in $|\log \epsilon|$ with period $2 \log c$. Substituting this flux density into (6), which involves an integral over $\partial \Omega$, yields $R \sim$ const $\times \epsilon^{b} e^{-\Delta W / \epsilon} G(|\log \epsilon|)$, where the exponent $b \equiv q-1$, and the function $G(\bullet)$ must have period $2 \log c$. This is the promised oscillatory rate formula. Interestingly, $b$ varies continuously as the model is changed.

Discussion.-The slowly oscillating factor $G(|\log \epsilon|)$ is related to a phenomenon discussed elsewhere [3]. If the noise strength $\epsilon$ is small, escape across a quadratic barrier follows the formally most probable escape path (the MPEP) only until it gets within an $\mathcal{O}\left(\epsilon^{1 / 2}\right)$ distance of the barrier. Thereafter escape occurs diffusively, rather than ballistically. Since the MPEP in the models considered here spirals geometrically into the barrier $\partial \Omega$, the point at which it gets within an $\mathcal{O}\left(\epsilon^{1 / 2}\right)$ distance will cycle around $\Omega$ as $\epsilon \rightarrow 0$, periodically in $|\log \epsilon|$. (Cf. Day [12]13].) In fact, the period will be $2 \log c$. If the effective diffusivity varies with position along $\partial \Omega$, one would expect the escape rate $R$ to be periodically modulated. That is what we have shown to occur.

We expect the phenomenon of slow oscillations is relevant to stochastic resonance in multistable continuous systems. Hu Gang et al. [18] have recently considered such systems, with the addition of time-periodic forcing and external noise. Steady states are then periodic attractors, separated by unstable limit cycles. In the weaknoise limit, the rate of noise-induced transitions should therefore include an oscillatory factor.

This research was partially supported by the National Science Foundation under grants NCR-90-16211 and DMS-95-00792 (RSM), and by the U.S. Department of Energy under contract DE-FG03-93ER25155 (DLS).

[1] Theory of Noise-Induced Processes in Special Applications, edited by F. Moss and P. V. E. McClintock (Cambridge Univ. Press, 1989); P. Hänggi, P. Talkner, and M. Borkovec, Rev. Mod. Phys. 62, 251 (1990).

[2] T. Naeh, M. M. Kłosek, B. J. Matkowsky, and Z. Schuss, SIAM J. Appl. Math. 50, 595 (1990).

[3] R. S. Maier and D. L. Stein, Phys. Rev. E 48, 931 (1993). Available as chao-dyn/9303017.

[4] R. S. Maier and D. L. Stein, Phys. Rev. Lett. 71, 1783 (1993); J. Statist. Phys. 83, 291 (1996). Available as cond-mat/9506097.

[5] P. Talkner, Z. Phys. B 68, 201 (1987).

[6] B. Gaveau et al., Phys. Rev. A 46, 825 (1992); I. L'Heureux and R. Kapral, Phys. Lett. A 136, 472 (1989); P. Rehmus, W. Vance, and J. Ross, J. Chem. Phys. 80, 3373 (1984).

[7] M. I. Dykman and M. A. Krivoglaz, Soviet Phys. JETP 
50, 30 (1979).

[8] M. I. Dykman et al., Nuovo Cimento D 17, 661 (1995).

[9] R. Graham and T. Tél, Phys. Rev. Lett. 52, 9 (1984).

[10] R. Graham and T. Tél, Phys. Rev. A 31, 1109 (1985).

[11] M. V. Day, in Diffusion Processes and Related Problems in Analysis, edited by M. Pinsky (Birkhäuser, 1990).

[12] M. V. Day, Stochastics 48, 227 (1994).

[13] M. V. Day, Virginia Tech preprint (unpublished).

[14] H. A. Kramers, Physica 7, 284 (1940).

[15] M. I. Freidlin and A. D. Wentzell, Random Perturbations of Dynamical Systems (Springer-Verlag, 1984).

[16] M. I. Dykman et al., Phys. Rev. Lett. 68, 2718 (1992).

[17] M. I. Dykman, M. M. Millonas, and V. N. Smelyanskiy, Phys. Lett. A 195, 53 (1994). Available as condmat/9410056.

[18] Hu Gang, A. Daffertshofer and H. Haken, Phys. Rev. Lett. 76, 4874 (1996). 